\title{
Nature de la flore microbienne dominante et sous-dominante des laits crus très pollués
}

\author{
par \\ J. RICHARD \\ Avec la collaboration de Chantal HOUSSU
}

\section{Rés u m é}

Pour étudier la nature de la flore microbienne dominante et sous dominante du lait cru très pollué (entre $10^{5}$ et $10^{8}$ microorganismes $/ \mathrm{ml}$ ), la flore totale et la flore résistante à la pénicilline de 231 échantillons de diverses origines (lait de traite manuelle ou mécanique, refroidi ou non à la ferme) ont été évaluées par ensemencement à la surface de la gélose à l'aide d'un appareil « Spiral ».

A partir des boîtes de dénombrement de la flore totale, on a prélevé 25 grosses colonies par échantillon et, lorsqu'elles étaient en flore dominante ou sous dominante, 8 petites colonies. Les premières ont été caractérisées à l'aide de 4 critères d'intérêt technologique (croissance au froid, lipolyse, protéolyse, production d'acide à partir du lactose) et de 10 à 12 critères taxonomiques; les secondes sur la base de 6 critères choisis pour identifier les streptocoques les plus intéressants. Une collection de 182 souches représentatives des grosses colonies résistantes à la pénicilline, a fait l'objet d'une classification numérique sur la base de 32 critères biochimiques et physiologiques.

Les grosses colonies ont été séparées en deux groupes sur la base de leur résistance à la pénicilline. Celles qui étaient résistantes (les plus nombreuses) étaient composées principalement de bactéries coliformes et de Pseudomonas du groupe fluorescent. Les colonies sensibles comprenaient aussi des bactéries à Gram négatif, mais surtout des microcoques et des bactéries corynéformes; dans l'ensemble elles présentaient des caractères technologiques peu

Laboratoire de Microbiologie Laitière et de Génie Alimentaire, I.N.R.A. - C.N.R.Z. 78350 Jouy-en-Josas (France). 
marqués. Les petites colonies étaient le plus souvent des streptocoques dont la moitié identifiée à $S$. lactis-cremoris.

Aucune compétition entre la flore lactique et la flore nuisible (Pseudomonas ou bactéries coliformes) n'a été mise en évidence.

Mots clés :

Lait cru - Flore microbienne - Composition.

Titre abrégé :

Nature de la flore du lait cru très pollué.

\section{Su m m a ry}

\section{COMPOSITION OF DOMINANT AND SUBDOMINANT FLORA OF MILK OF POOR BACTERIOLOGICAL QUALITY}

The composition of the dominant and subdominant microbial flora of raw milk produced under different conditions (hand or machine milking, milk storage at three ranges of temperature i.e. $<5^{\circ} \mathrm{C}, 8-12^{\circ} \mathrm{C}, \geqslant 15^{\circ} \mathrm{C}$ for 1 or 2 days) has been studied at 3 periods in the course of one year (spring, summer and autumn).

Samples dilutions (1:50 in quarter strength Ringer solution) were made and mechanically shaken to break down the clumps before they were surface plated on Plate count Agar (PCA) and Nutrient Agar containing $100 \mathrm{IU} / \mathrm{ml}$ penicillin, using a Spiral Plater. After incubation for $1-2 d$ at $30^{\circ} \mathrm{C}$ plus 2-3 $d$ at room temperature the large $(>2 \mathrm{~mm})$ and the «small» $(\leqslant 2 \mathrm{~mm})$ colonies on PCA were counted using a laser counter adapted to the Spiral plating.

From each plate for total count 25 large and, when ever possible, 8 small well isolated colonies were picked at random and without any purification transferred on various test media for primary characterization; large colonies were submitted to 10-16 tests; these were chosen to demonstrate technological properties of isolates (growth at low temperature, lipolysis, proteolysis, production of acid from lactose) and included taxomonical tests easy to perform on a large scale. The criteria for small colonies were those in use for the identification of the lactic streptococci and the separation of the other streptococci into genus or groups. A representative collection of 182 penicillin resistant bacteria was purified and submitted to an adansonian classification based on 32 physiological and biochemical criteria including thoses for primary characterization. $A$ set of 7 criteria was derived from this step of the study to try to identify the remaining isolates of penicillin resistant bacteria.

On the whole, 4810 large colonies from 231 milk samples were characterized. Most of them were resistant to penicillin; in this group coliform bacteria and Pseudomonas (fluorescent group) were 
dominant Acinetobacter spp. and yellow pigmented bacteria allocated to Cytophaga-Flavobacter group were present at a lower level. The penicillin sensitive bacteria were micrococci, coryneform bacteria and some coliform and yellow pigmented bacteria, the whole showing a weak biochemical pattern as compared to Pseudomonas and coliform bacteria. Most of the small colonies (1424 strains examined from 178 samples) were streptococci, about $50 \%$ being identified as S. lactis-cremoris. The genus Leuconostoc was present at a significant level (10-20\%) only in autumn; fecal streptococci were at a very low level.

No evidence of a competition between the lactic acid bacteria and the Pseudomonas counts in the milk was observed. It was concluded that the lactic streptococci had no action on the undesirable flora of the raw milk, probably because they did not reach a sufficient level of population.

Key words:

Raw milk - Bacteriological flora - Composition.

\section{INTRODUCTION}

Il n'existe pas dans la littérature scientifique d'étude de la flore microbienne du lait cru à la fois précise sur le plan taxonomique (identification ou description suffisante des micro-organismes) et valable sur le plan statistique (précision des conditions de production et de conservation du lait, nombre et représentativité satisfaisants des échantillons).

Abd-el-Malek et Gibson (1948, 1952), par exemple, ont sans doute été les premiers à faire une étude précise de la flore microbienne $\mathrm{du}$ lait $\mathrm{cru}$ et sur ses caractéristiques physiologiques, biochimiques et technologiques, mais ils ne donnent pas la fréquence des différents micro-organismes identifiés, ni précisément l'origine des échantillons. Thomas et al., (1962) étudiant la flore de lait (certainement conservé sans refroidissement) ont montré que les microcoques dominent dans le lait peu contaminé $\left(<2 \times 10^{4}\right.$ micro-organismes $/ \mathrm{ml}$ ); le pourcentage de streptocoques varie de 10 à 20 quelle que soit la population microbienne, alors que la flore à Gram négatif passe de $6 \%$ à $54 \%$ lorsque la flore totale passe de $5 \times 10^{3}$ à $5 \times 10^{6} / \mathrm{ml}$. Ils ne précisent pas la nature des streptocoques trouvés ni celle des bactéries à Gram négatif. Twomey et Crawley (1968) ont fait une étude similaire qui confirme les résultats précédents. Ils ont montré que les streptocoques ne représentent qu'un faible pourcentage de la flore (moins de $5 \%$ ), tant qu'elle reste au-dessous de 200000 micro-organismes $/ \mathrm{ml}$. Les streptocoques ( $S$. lactis-cremoris) représentent cependant environ $1 / 4$ de la flore du lait des échantillons (4 seulement) qu'ils ont examinés 
dans la gamme de 200000 à 400000 micro-organismes $/ \mathrm{ml}$. On sait donc finalement peu de choses de la nature et des propriétés d'intérêt technologique de la flore du lait très pollué du fait d'un refroidissement insuffisant ou de la malpropreté de la vaisselle laitière.

On a plus d'information par contre sur la flore psychrotrophe du lait cru (Witter 1961, Thomas et Thomas 1973). Cette flore est essentiellement composée de bactéries à Gram négatif productrices de lipases et de protéases jugées responsables des défauts de goût des fromages (Vassal et Auclair 1966; Mourgues et al., 1967). Cependant, dans une étude récente, nous avons montré que seule une petite fraction de cette flore est capable de se développer rapidement dans le lait cru conservé à moins de $5^{\circ} \mathrm{C}$ (Richard 1981 a). Ces bactéries, identifiables à Pseudomonas fluorescens, constituent l'essentiel de la flore dominante lorsque le lait contient, après 5 jours de conservation à $5^{\circ} \mathrm{C}$, plus de $10^{6}$ micro-organismes $/ \mathrm{ml}$ (Richard, 1981 b).

A la demande de professionnels laitiers, nous avons entrepris une étude de la flore dominante du lait cru refroidi ou non, produit dans une zone où l'on trouvait encore des échantillons contenant plus de $10^{6}$ micro-organismes $/ \mathrm{ml}$. Nous en avons profité pour essayer de mettre en évidence, soit un antagonisme entre la flore lactique naturelle et la flore nuisible (protéolytique et/ou lipolytique), soit l'existence de conditions de traite et/ou de conservation du lait plus favorables à la première qu'à la seconde.

Une méthode inspirée de celle de Corlett et al., (1965), de Vanderzant et Patel (1967) et de Gyllenberg (1963) a été mise en œuvre afin de permettre l'identification à un niveau acceptable d'un grand nombre de souches ou tout au moins, de les caractériser d'un point de vue technologique.

\section{METHODES}

\section{Origine des échantillons}

Les échantillons de lait cru examinés ont été prélevés dans un laboratoire interprofessionnel parmi ceux qui servaient à l'analyse en vue du paiement du lait à la qualité. Ils ont été choisis en fonction des origines suivantes :

- mode de traite : mécanique ou manuelle;

- température de conservation du lait : non refroidi (conservation à température ambiante), refroidi entre 8 et $12^{\circ} \mathrm{C}$, refroidi à moins de $5^{\circ} \mathrm{C}$;

- durée de conservation à la ferme : 1 ou 2 jours.

Les prélèvements ont été effectués aux quatre périodes de l'année suivantes :

- Série I : au printemps, avant la mise à l'herbe. 
- Série II : au printemps, après la mise à l'herbe.

- Série III : à l'automne, avant la rentrée des animaux.

- Série IV : à l'automne, après la rentrée des animaux.

\section{Dénombrements microbiens}

On a effectué, dans le laboratoire interprofessionnel, les dénombrements microbiens suivants par la méthode d'ensemencement en spirale* à la surface de la gélose (Gilchrist et al., 1972) après dilution du lait à 1/50 dans l'eau de Ringer diluée au quart et traitement du liquide de dilution 30 secondes à l'Ultra-Turrax pour disperser les amas microbiens (Richard, 1980) :

- Flore totale sur milieu PCA (Plate Count Agar Difco 479) additionné, à partir de la série II, de $1 \%$ de lait écrémé ;

- Flore pénicillino-résistante mésophile sur milieu NA (Nutrient Agar Difco 1) additionné de $100 \mathrm{Ul} / \mathrm{ml}$ de pénicilline G.

Les boîtes de Petri ont été incubées au laboratoire interprofessionnel pendant $24 \mathrm{~h}$ à $48 \mathrm{~h}$ à $30^{\circ} \mathrm{C}$, puis envoyées à notre laboratoire par colis S.N.C.F. (2 à 3 jours de voyage à température ambiante).

Le dénombrement des colonies a été fait dans notre laboratoire à l'aide d'un compteur électronique à laser adapté à l'ensemencement en spirale. Les petites colonies (diam. $\leqslant 2 \mathrm{~mm}$ ) ont été comptées séparément (Richard, 1982).

\section{Caractérisation primaire de la flore}

Après dénombrement des colonies on a choisi un nombre de boîtes représentatif des diverses catégories de lait. Sur les boîtes de dénombrement de la flore totale, on a prélevé, au hasard, parmi la flore dominante (colonies en périphérie), 25 grosses colonies et/ou 8 petites colonies (diam. $\leqslant 2 \mathrm{~mm}$ ). Une suspension dans l'eau stérile d'un fragment des grosses colonies a été repiquée à l'aide d'un appareil multipoint sur des milieux gélosés appropriés, à raison de 25 colonies par boîte pour mettre en évidence les caractères suivants :

- Résistance à $100 \mathrm{U} / \mathrm{ml}$ de pénicilline G (milieu PCA).

- Croissance sur milieu VRB (Violet Red Bile Agar, Difco 12).

- Production d'acide à partir du lactose sur VRB et/ou sur milieu non sélectif (Red Phenol Base, Difco )gélosé et additionné de $0,5 \%$ de lactose.

- Lipolyse : hydrolyse de la tributyrine émulsifiée par un détergent non ionique (Triton X 100) et incorporé au PCA à raison de $1 \%$.

\footnotetext{
* Inter-Science, 3, rue de Milan - 75009 Paris.
} 
- Protéolyse sur milieu PCA additionné de 0,2\% de gélatine, mise en évidence par le chlorure mercurique en milieu chlorhydrique.

- Croissance à $2^{\circ} \mathrm{C}$, à $5^{\circ} \mathrm{C}$ et à $40^{\circ} \mathrm{C}$ sur $\mathrm{PCA}$.

- Production d'un pigment fluorescent sur milieu P (Difco) avec examen des boîtes en lumière ultra violette.

- Présence d'une oxydase par une méthode rapide (Gaby et Hadley, 1957).

- Culture sur PCA en anaérobiose (jarres anaérobies Gaspak $\mathrm{H} 2+\mathrm{CO} 2 \mathrm{BBL})$.

A titre d'essai, on a utilisé, en plus, les critères suivants :

- Culture sur PCA à pH4 (série I et II) et à pH4,5 (série I).

- Fluorescence sur milieu F (Difco) (série I).

- Culture à $10^{\circ} \mathrm{C}$ (série II).

- Catalase (série III et IV).

- Culture sur PCA contenant $40 \mathrm{mg}$ d'acide nalidixique par litre (série IV).

Ces critères ont été choisis pour permettre un classement des micro-organismes isolés en fonction de leur intérêt technologique (croissance à basse température, protéolyse, lipolyse, acidification à partir du lactose) et un classement à caractère taxonomique de la manière qui sera indiquée plus loin.

Les petites colonies non pigmentées, supposées être des streptocoques, ont été soumises aux critères de base suivants :

1. morphologie à l'état frais après $24 \mathrm{~h}$ de culture sur bouillon Trypticase Soja (Difco) réparti à raison de $0,2 \mathrm{ml}$ dans les cupules d'une plaque de microtitration stérile,

2. catalase sur ce dernier et sur PCA,

3 et 4 . culture à 10 et $45^{\circ} \mathrm{C}$,

5. culture en présence de $6,5 \%$ de $\mathrm{Na} \mathrm{Cl}$,

6. $\mathrm{pH}$ atteint en $48 \mathrm{~h}$ à $30^{\circ} \mathrm{C}$ sur bouillon APT (Difco) additionné de $1 \%$ de glucose et réparti dans des clochettes de Durham (2 $\mathrm{ml} /$ tube),

7. présence, dans ce bouillon, de gaz révélé en traitant les tubes 5 à 10 secondes aux ultra-sons $(80 \mathrm{k} \mathrm{Hz})$.

Les tests 3 à 5 ont été exécutés sur PCA additionné de $1 \%$ de lait écrémé stérile.

\section{Classification numérique et tentative d'identification}

Sur l'ensemble des colonies résistantes à la pénicilline, isolées au cours de la série I, nous avons sélectionné 84 souches parmi les groupes technologiques les plus représentatifs. Une collection 
de 112 souches représentatives des grosses colonies (résistantes ou non à la pénicilline) isolées au cours des séries II et III a également été constituée. Les micro-organismes ont fait l'objet d'une classification numérique sur la base des critères suivants :

a) Utilisation, comme seule source de carbone, de 7 composés organiques : acétate, arginine, citrate, glucose, inositol, lactose, tréhalose, le milieu de base étant de la gélose ordinaire additionnée de sels minéraux (Stanier et al., 1966).

b) Culture sur PCA à $\mathrm{PH} 4,0$ et 4,5 ou en présence de chlorure de sodium $(4$ et $6,5 \%)$ ou à $2^{\circ} \mathrm{C}, 5^{\circ} \mathrm{C}$ et $40^{\circ} \mathrm{C}$ ou sur milieu SS Agar (Difco, 74), VRB Agar (Difco, 12), Selenite Broth (Difco, 275) et Malonate Broth (Difco, 395) tous deux additionnés de $15 \mathrm{~g} / 1$ de gélose ou sur PCA en anaérobiose (gaz Pack BBL).

c) Hydrolyse de l'amidon (milieu PCA + 1\% d'amidon, mise en évidence par le lugol), de la gélatine (milieu PCA $+0,2 \%$ de gélatine, mise en évidence par une solution de chlorure mercurique en milieu chlorhydrique), de la tributyrine (milieu PCA + $2 \%$ d'une émulsion à $50 \%$ de tributyrine dans de l'eau contenant $2 \%$ de Triton X 100), du Tween 80 sur milieu de Colwell et Wiebe (1970).

d) Recherche d'une arginine dihydrolase (Thornley, 1963), de l'oxydase (Gaby et Hadley, 1957), de la nitrate-réductase, de l'ornithine décarboxylase et réaction de Voges-Proskauer (Harrigan et Mc Cance, 1976).

e) Formation d'un pigment fluorescent sur milieux $\mathrm{P}$ et $\mathrm{F}$ (milieux Difco) avec examen des colonies en lumière UV.

f) Morphologie et étude de mobilité par la technique des gouttes pendantes (Lowis, 1969).

g) Coloration de Gram.

h) Voie du métabolisme du glucose par la technique à un tube (Park et Holding, 1966).

Après purification, les colonies ont été cultivées 24 à 48 h sur milieu NA puis une suspension de chaque souche contenant $10^{4}$ à $10^{5}$ cellules $/ \mathrm{ml}$ d'eau stérile a été préparée. Les suspensions ont servi à incuber les différents milieux ci-dessus à raison de 25 souches/boîte.

Tous les tests, sauf le métabolisme du glucose, ont été codés de la façon suivante :

- 00 : résultat négatif ou douteux;

- 10 : résultat positif faible;

- 11 : résultat positif intense;

Le pourcentage de similitude entre souches prises 2 à 2 a été calculé suivant la formule de Sokal et Michener et celle de Jaccard (Sokal et Sneath, 1963) puis les souches ont été classées en fonction de leur ressemblance suivant le critère du saut minimal (Benzecri, 1975). Nous avons ainsi formé des groupes dans lesquels les souches 
ont environ $70 \%$ de similitude interne. Ce seuil correspond, compte tenu des critères utilisés, soit au niveau du genre, soit au niveau de l'espèce (Sokal et Sneath). Enfin, on a tenté une identification de deux à trois micro-organismes appartenant aux groupes les plus importants numériquement en prenant le Bergey's Manual (1974) comme référence, sauf dans la série I où les coliformes ont été identifiés à l'aide de galeries API 20E*.

\section{RESULTATS}

\section{Nature de la flore dominante}

a) Classification numérique et identification des souches représentatives des grosses colonies

La figure 1 montre, sous forme d'un dendrogramme simplifié, la relation entre les souches pénicillino-résistantes de la série I. Trois groupes principaux à $68-70 \%$ de similitude se dégagent :

- le groupe $A$ comprenant 19 souches de bactéries coliformes appartenant à plusieurs espèces ;

- le groupe $B$ constitué par le sous-groupe $\mathrm{B} 1$, relativement homogène, composé de 8 bactéries identifiables au genre Acinetobacter, le sous-groupe B2 comprenant 2 bactéries pigmentées jaunes (groupe Flavobacter-Cytophaga) et le sous-groupe B3 rassemblant diverses bactéries Gram-négatif non identifiées;

- le groupe $C$, comprenant à lui seul près de la moitié des souches, identifiables aux Pseudomonas du groupe P.fluorescens (Richard, 1981).

Une structure analogue a été obtenue après classement des souches pénicillino-résistantes isolées au cours des séries II et III (fig. 2). A noter toutefois que les bactéries coliformes formaient deux groupes séparés, l'un composé de 8 souches protéolytiques et lipolytiques, l'autre comprenant 28 souches n'ayant aucun de ces caractères. L'identification de représentants de ces bactéries au niveau de l'espèce à l'aide des critères retenus dans le Manuel de Bergey a posé des problèmes compte tenu de la grille rigide que nous avons adoptée : la plupart des souches différaient du type par 2 à 3 critères. Cependant, dans l'ensemble, les souches protéolytiques et lipolytiques ressemblaient à Ent.aerogenes, Hafnia alvei ou au genre Serratia et les autres à Ent.aerogenes, Ent.cloacae ou au genre Klebsiella. Cette collection comprenait en outre trois bactéries identifiables au genre Aeromonas, et 17 souches pour la plupart sensibles à la pénicilline, inclassables car ayant donné des résultats

* A.P.I. System, La Balme-les-Grottes - 38390 Montalieu-Vercieu. 


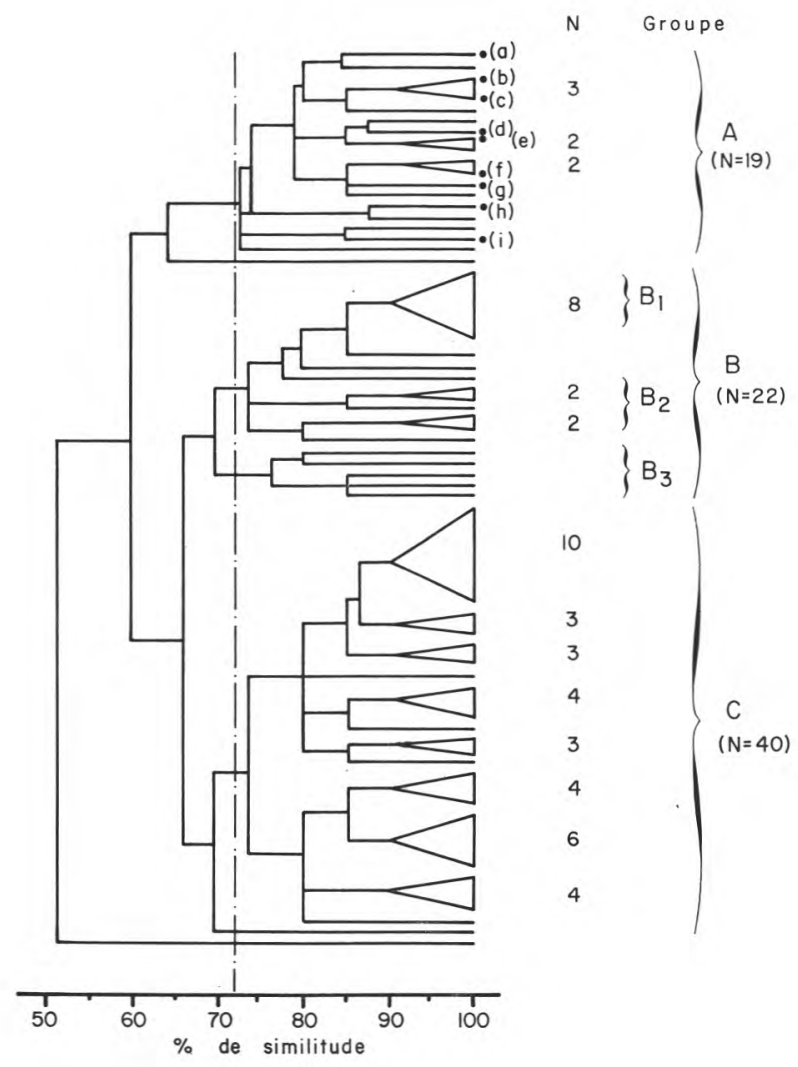

Fig. 1

Classification, suivant les indices de Sokal et Michener et la méthode d'agrégation par saut minimum, des 84 souches de bactéries résistantes à la pénicilline isolées au cours de la série I (dendrogramme simplifié).

A : bactéries coliformes $; \mathrm{B}_{1}$ : Acinetobacter $; \mathrm{B}_{2}$ : groupe complexe comprenant 2 souches jaune (groupe Cytophaga-Flavobacter); C : Pseudomonas.

(a) et (f) : Citrobacter freundii; (b) et (c) : Hafnia alvei; (d) et (c) : Klebsiella pneumoniase; (g) Enterobacter cloacae; (h) : Serratia sp.; (i) Erwinia herbicola. $\mathrm{N}$ : nombre de souches dans les groupes constitués à environ $90 \%$ de similitude.

Numerical classification of penicillin resistant bacteria (84 strains) isolated from milk in series I (simple matching coefficients, single linkage clustering, simplified dendrogram).

A: Coliform bacteria; $B_{1}$ : Acinetobacter $s p p ; B_{2}$ : complex group comprising 2 yellow strains (Cytophaga-Flavobacter group); $C:$ Pseudomonas spp. 


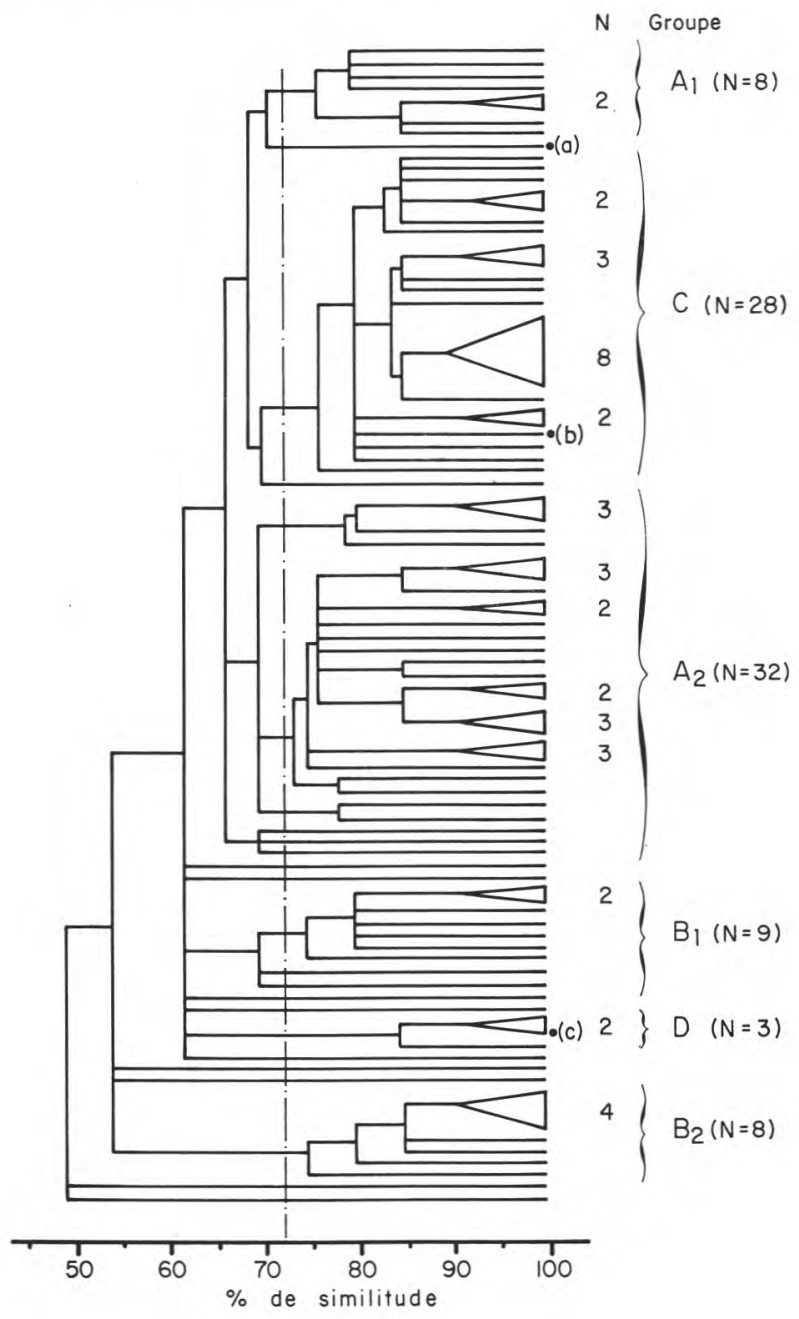

Fig. 2

Classification, suivant les indices de Jaccard et la méthode d'agrégation par saut minimum, des 98 souches résistantes à la pénicilline isolées au cours des séries II et III (dendrogramme simplifié).

$A_{1}$ : bactéries coliformes psychrotrophes ;

$\mathrm{A}_{2}$ : bactéries coliformes mésophiles ;

$\mathrm{B}_{1}$ : Acinetobacter

$\mathrm{B}_{2}$ : Groupe Cytophaga-Flavobacter;

$\mathrm{C}$ : Pseudomonas.

(a) et (b) : Vibrionaceae; (c) : Aeromonas hydrophila (identification par galerie API $20 \mathrm{E}$ ).

Numerical classification of penicillin resistant bacteria (98 strains) isolated from milk in series II and III (summer) (coefficients of Jaccard, single linkage clustering, simplified dendrogram).

$A_{1}$ : psychrotrophic coliform bacteria;

$A_{2}$ : mesophilic coliform bacteria;

$B_{1}$ : Acinetobacter spp.;

$B_{2}$ : Cytophaga-Flavobacter group;

$C$ : Pseudomonas. 
TABLEAU 1 - TABLE 1

Caractéristiques intéressantes des principaux groupes de bactéries résistant à la pénicilline (résultats extraits des tests exécutés pour la classification numérique)

Some characters of taxonomical interest for penicillin resistant bacteria (tests selected after numerical classification)

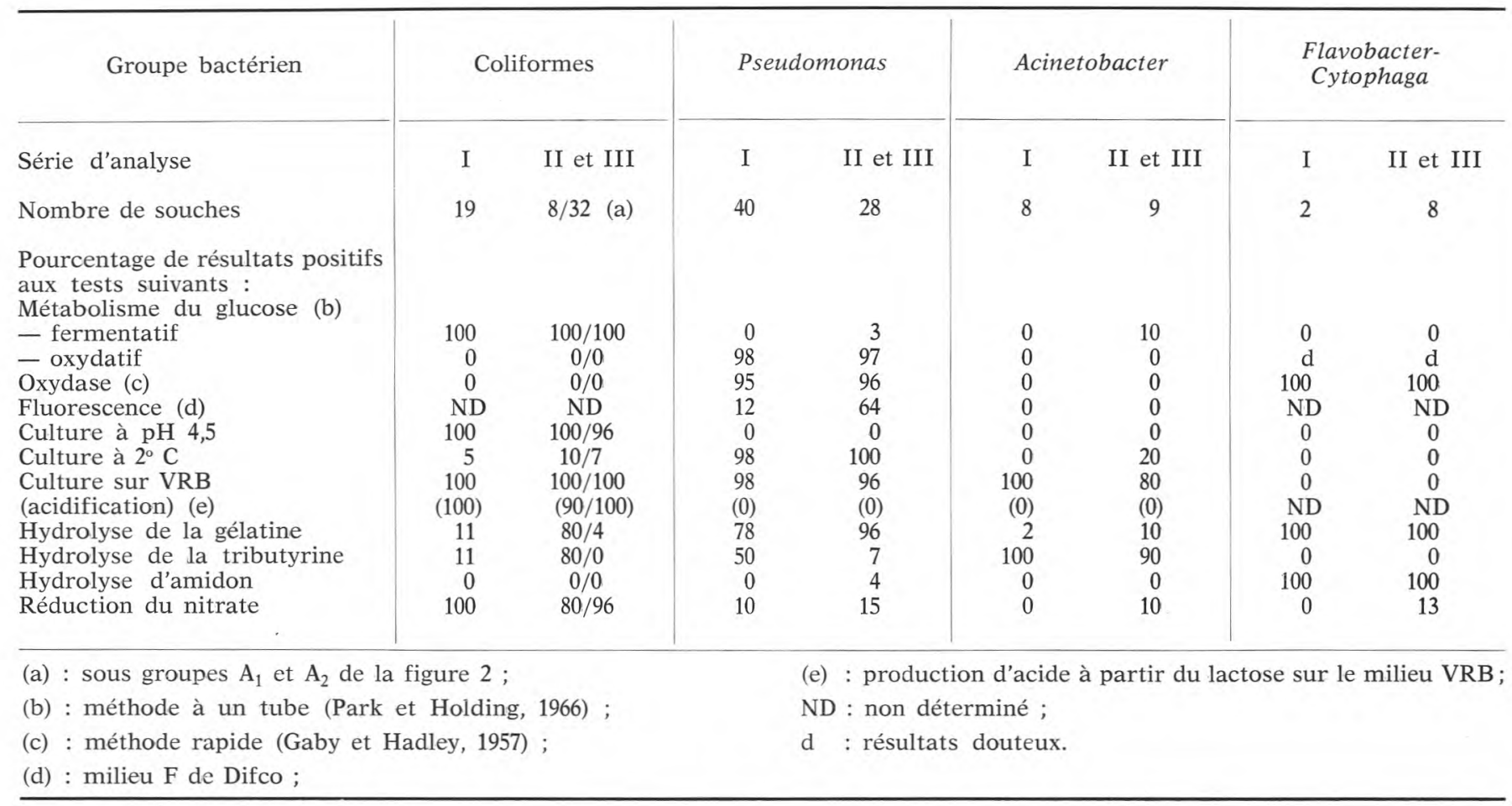


négatifs à la plupart des tests. A l'examen microscopique ces souches avaient l'aspect de microcoques ou de bactéries corynéformes.

Le tableau 1 montre les caractères les plus intéressants pour départager les principaux groupes de bactéries résistantes à la pénicilline. Toutes les souches classées comme Pseudomonas n'étaient pas oxydase-positives. Cela est dû à ce que la méthode rapide utilisée est un peu moins sensible que la méthode de référence (Richard, 1981 b). On notera aussi qu'un faible pourcentage de souches classées dans ce genre révélait un métabolisme fermentatif. Il s'agissait certainement de bactéries du genre Aeromonas. Il est remarquable également qu'un faible pourcentage de souches réduisait le nitrate à l'exception des bactéries coliformes pour lesquelles ce caractère est normalement positif (Cowan, 1974).

b) Classification finale de l'ensemble des grosses colonies résistantes à la pénicilline

$\mathrm{Au}$ total, 4810 grosses colonies prélevées à partir de 231 échantillons de lait ont été étudiées sur la base des 10 à 16 critères d'intérêt technologique et/ou taxonomique décrits plus haut.

L'appartenance des souches résistantes à la pénicilline aux 4 groupes principaux dégagés pour l'étude taxonumérique a été déterminée sur la base des caractères suivants extraits du tableau 1 :

TABLEAU 1

\begin{tabular}{|c|c|c|c|c|}
\hline & Coliformes & Pseudomonas & Acinetobacter & $\begin{array}{l}\text { Flavobact. } \\
\text { Cytophaga }\end{array}$ \\
\hline Pigmentation & - & - & - & $\begin{array}{c}\text { Colonies } \\
\text { jaunes }\end{array}$ \\
\hline $\begin{array}{l}\text { Oxydase } \\
\text { Anaérobiose } \\
\text { Culture sur VRB } \\
\text { Attaque du lactose (VRB) } \\
\text { Protéolyse } \\
\text { Lipolyse }\end{array}$ & $\begin{array}{l}- \\
+ \\
+ \\
+ \\
(\ldots) \\
(\ldots)\end{array}$ & $\begin{array}{l}+ \\
+ \\
+ \\
+ \\
(\ldots)\end{array}$ & $\begin{array}{l}- \\
\overline{(\ldots)} \\
\overline{-} \\
+\end{array}$ & $\begin{array}{l}+ \\
- \\
(\ldots) \\
+ \\
-\end{array}$ \\
\hline
\end{tabular}

(...) Caractères non pris en considération (variables ou non déterminés).

Cette classification a été confirmée ou complétée selon les séries, pour les caractères suivants : culture à $2^{\circ} \mathrm{C}$, à $\mathrm{pH} 4,5$, sur milieu SS, production d'un pigment fluorescent sur milieu $\mathrm{F}$ ou P.

Suivant les séries, 62 à $100 \%$ des souches ont pu ainsi être classées dans l'un des 4 groupes définis plus haut. 

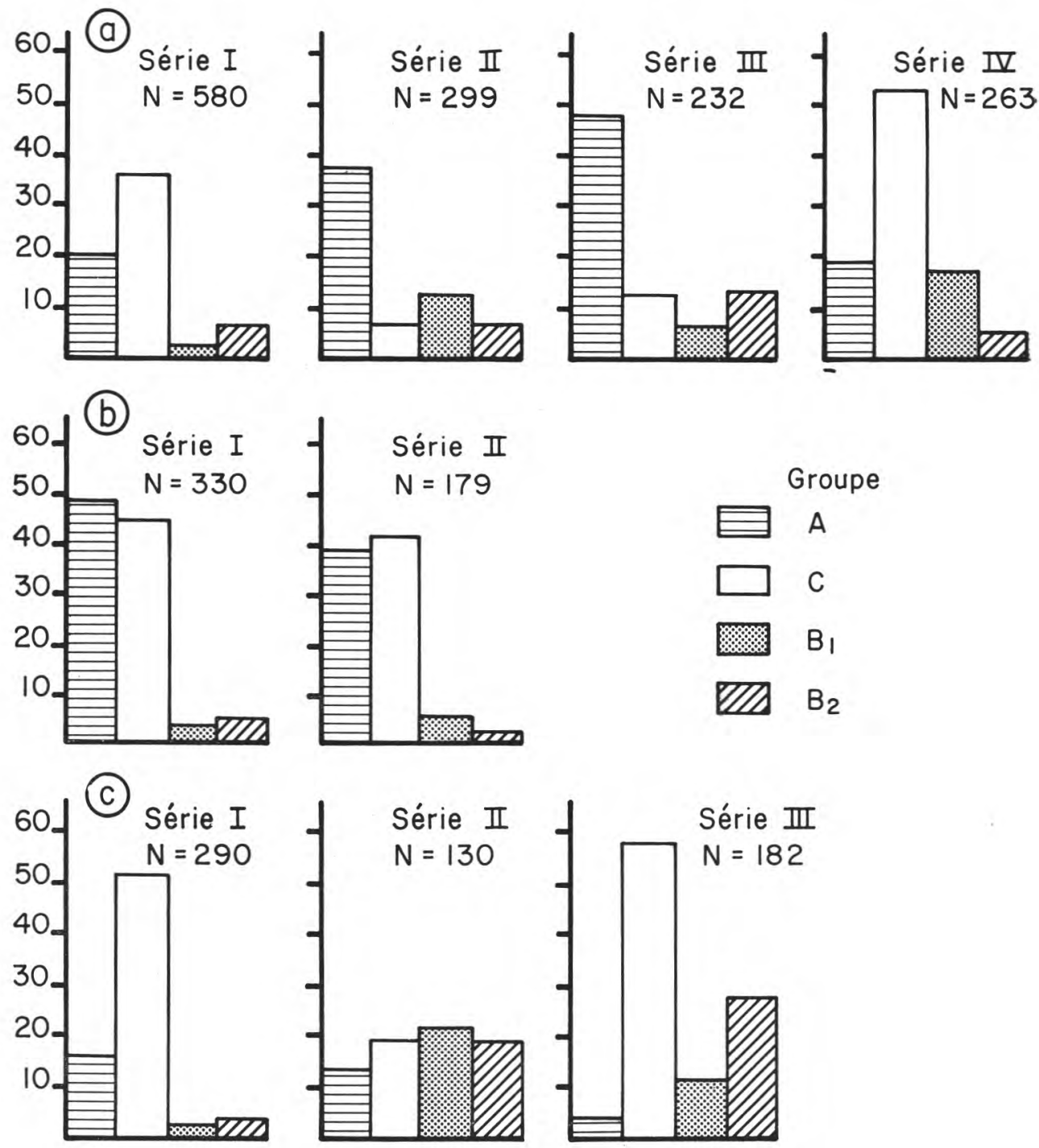

Fig. 3

Pourcentage (en ordonnée) de bactéries coliformes (groupe A), de Pseudomonas (groupe C), d'Acinetobacter (groupe $\mathrm{B}_{1}$ ) et de Flavobacter-Cytophaga (groupe $\mathrm{B}_{2}$ ) dans la flore pénicillino-résistante. $\mathrm{N}=$ nombre de souches ; (a), (b) et (c) : conservation du lait respectivement à température ambiante, entre 8 et $12^{\circ} \mathrm{C}$, à moins de $5^{\circ} \mathrm{C}$.

Percentage in penicillin resistant flora of coliform bacteria (A), Pseudomonas $(C)$, Acinotobacter spp. $\left(B_{1}\right)$ et Flavobacter-Cytophaga group $\left(B_{2}\right) ;(a),(b),(c)$ milk storage at respectively room temperature, between 8 and $12^{\circ} C$, less than $5^{\circ} C$. 
TABLEAU $2-T A B L E 2$

Caractères d'intérêt technologique de la flore non lactique sensible à la pénicilline

Characters of technological interest of the non lactic penicillin sensitive bacteria

\begin{tabular}{|c|c|c|c|c|c|c|}
\hline & \multirow{2}{*}{ Culture à } & \multirow{2}{*}{$\begin{array}{l}\text { Nombre de } \\
\text { souches }\end{array}$} & \multicolumn{4}{|c|}{ Pourcentage de souches } \\
\hline & & & Acidifiantes (a) & Protéolytiques (b) & \multicolumn{2}{|c|}{ Lipolytiques (c) } \\
\hline Série II & $\begin{array}{r}2^{\circ} \mathrm{C} \\
5^{\circ} \mathrm{C} \\
5^{\circ} \mathrm{C}\end{array}$ & $\begin{array}{r}38 \\
137 \\
199\end{array}$ & $\begin{array}{l}82 \\
44 \\
59\end{array}$ & $\begin{array}{l}11 \\
18 \\
12\end{array}$ & $\begin{array}{r}5 \\
42 \\
30\end{array}$ & $\begin{array}{r}8 \\
47 \\
36\end{array}$ \\
\hline Série III & $\begin{array}{r}2^{\circ} \mathrm{C} \\
5^{\circ} \mathrm{C} \\
>5^{\circ} \mathrm{C}\end{array}$ & $\begin{array}{r}49 \\
199 \\
367\end{array}$ & $\begin{array}{l}36 \\
17 \\
19\end{array}$ & $\begin{array}{l}10 \\
10 \\
24\end{array}$ & $\begin{array}{l}24 \\
37 \\
50\end{array}$ & $\begin{array}{l}28 \\
42 \\
71\end{array}$ \\
\hline Série IV & $\begin{array}{r}2^{\circ} \mathrm{C} \\
5^{\circ} \mathrm{C} \\
>5^{\circ} \mathrm{C}\end{array}$ & $\begin{array}{r}77 \\
175 \\
84\end{array}$ & $\begin{array}{l}56 \\
28 \\
44\end{array}$ & $\begin{array}{l}38 \\
39 \\
20\end{array}$ & $\begin{array}{r}5 \\
35 \\
2\end{array}$ & $\begin{array}{l}19 \\
53 \\
13\end{array}$ \\
\hline
\end{tabular}

(a) : milieu Red Phenol Base, Difco, gélosé à $15 \mathrm{~g} / 1+0,5 \%$ de lactose ; (b) : hydrolyse de la gélatine et (c) de la tributyrine (voir méthodes). 
La figure 3 montre la proportion de souches de chacun de ces groupes bactériens dans le lait de diverses origines. On constate que dans le lait conservé à plus de $15^{\circ} \mathrm{C}$, les Pseudomonas dominent en période froide (séries I et IV) alors que les bactéries coliformes dominent en période chaude (séries II et III); ces deux groupes sont à peu près à égalité dans le lait refroidi entre 8 et $12^{\circ} \mathrm{C}$ alors que les Pseudomonas dominent dans le lait conservé à moins de $5^{\circ} \mathrm{C}$. Les différences s'expliquent aisément par l'effet sélectif de la température du lait. D'une façon générale, les bactéries des groupes Acinetobacter et Flavobacter-Cytophaga sont nettement moins fréquentes que les Pseudomonas et les bactéries coliformes, ce qui est en accord avec les résultats de la classification des souches représentatives des séries I, II et III.

Le tableau 2 présente les caractéristiques d'intérêt technologique de la flore non lactique sensible à la pénicilline (toutes origines des échantillons confondues). Près de la moitié de cette flore n'était pas psychrotrophe, (absence de cuture sur PCA en 10 jours à $5^{\circ} \mathrm{C}$ ). Les souches protéolytiques l'étaient en général de façon moins active que les Pseudomonas (diamètre d'hydrolyse nettement plus faible). Plus de la moitié des souches étaient inhibées par le milieu destiné à mettre la lipolyse en évidence ; on ne sait donc pas si elles étaient lipolytiques ou non.

\section{c) Identification des petites colonies}

Le tableau 3 rassemble les résultats de l'étude des petites colonies. 178 échantillons ont été examinés. Environ $95 \%$ des colonies repiquées ont cultivé. Celles-ci comprenaient en moyenne $85 \%$ de souches à catalase négative, pour la plupart des streptocoques. (cocci par paires ou chaînette).

On a identifié les streptocoques les plus intéressants (et les plus fréquents) de la manière suivante (Sandine et al., 1972) :

TABLEAU 2

\begin{tabular}{|c|c|c|c|}
\hline Test & $\begin{array}{c}\text { Streptococcus } \\
\text { lactis - cremoris }\end{array}$ & $\begin{array}{c}\text { Genre } \\
\text { Leuconostoc }\end{array}$ & $\begin{array}{l}\text { Streptocoques } \\
\text { fécaux }\end{array}$ \\
\hline $\begin{array}{l}\text { Culture à } 10^{\circ} \mathrm{C} \\
\text { Culture à } 45^{\circ} \mathrm{C} \\
\mathrm{NaCl} \\
\mathrm{pH} \\
\mathrm{Gaz}\end{array}$ & $\begin{array}{l} \pm \\
\overline{4} \\
-\end{array}$ & $\begin{array}{l}(\ldots) \\
(\ldots) \\
(\ldots) \\
(\ldots) \\
+\end{array}$ & $\begin{array}{l}+ \\
+ \\
+ \\
+ \\
(\ldots) \\
-\end{array}$ \\
\hline
\end{tabular}

(...) Caractères non pris en considération. 

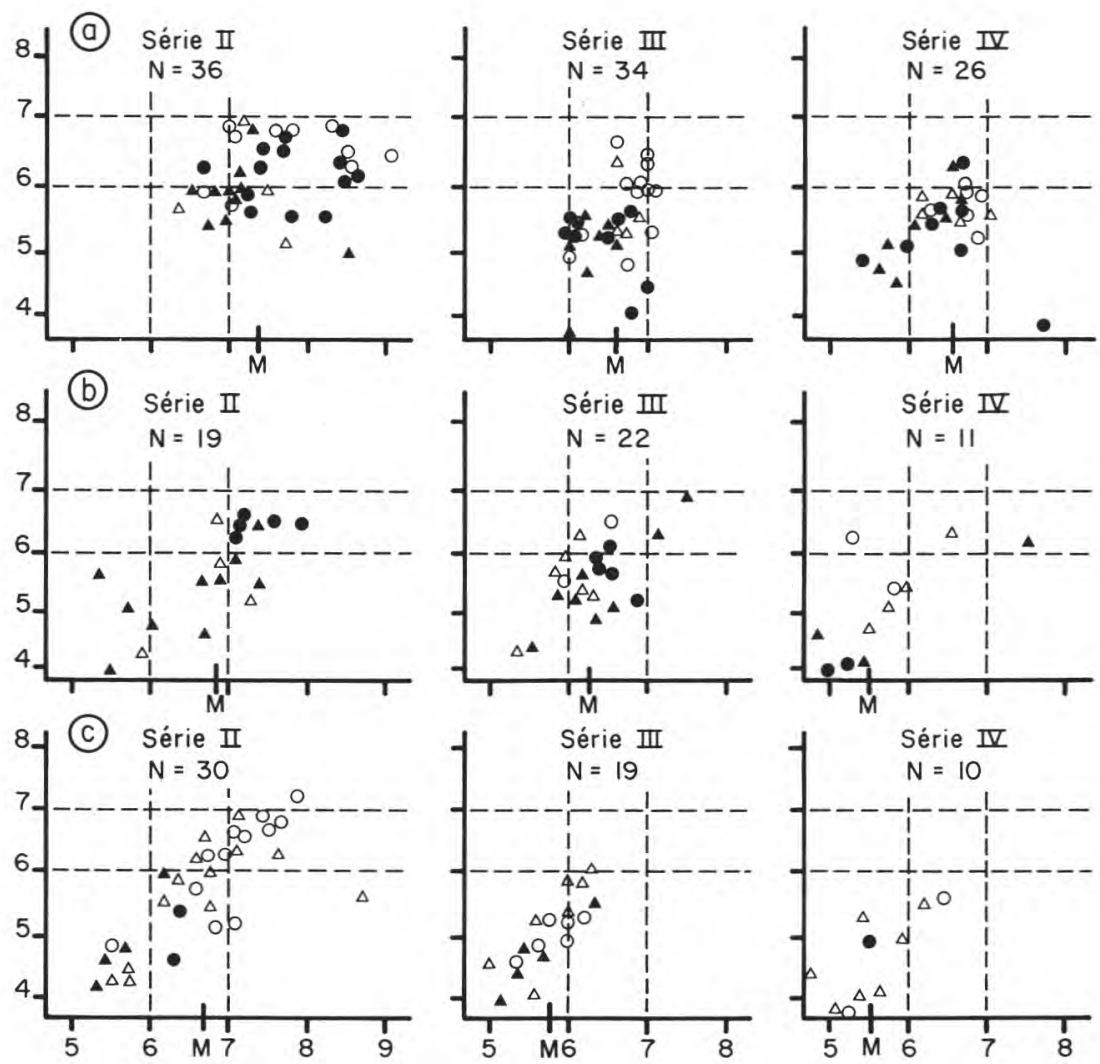

Fig. 4

Relation entre les logarithmes des nombres de bactéries résistantes à la pénicilline (en ordonnée) et des nombres de germes totaux (en abscisse) (a), (b) et (c) : conservation du lait respectivement à température ambiante, entre 8 et $12^{\circ} \mathrm{C}$, à moins de $5^{\circ} \mathrm{C}$.

$\Delta, \Delta$ : traite mécanique, resp. 1 ou $2 \mathrm{j}$ de conservation du lait.

- $\bigcirc$ : traite manuelle, resp. 1 ou $2 \mathrm{j}$ de conservation du lait.

M : médiane de la distribution, sur la base de la flore totale.

Relationship between the numbers of penicillin resistant bacteria $\left(l_{10}\right.$, vertical axis) and the total counts in milk ( $\lg _{10}$, abscissa) $(a),(b),(c)$ : as to Figure 3.

$\mathbf{\Lambda}, \triangle$ : machine milking, 1 and $2 d$ of storage resp.

- $\bigcirc$ : hand milking, 1 and $2 d$ of storage resp.

L'espèce S.lactis-cremoris représentait près des $2 / 3$ de ces souches, soit au total environ $55 \%$ des petites colonies, cela quelle que soit l'origine des échantillons ou la série. Les streptocoques fécaux étaient rares (moins de $5 \%$ ). Le genre Leuconostoc était 
TABLEAU 3 - TABLE 3

Etude des petites colonies dominantes ou sous-dominantes sur le milieu de dénombrement de la flore totale

Study of the small colonies present as predominant or subpredominant flora on the plates for total count

\begin{tabular}{|c|c|c|c|c|c|c|c|c|}
\hline \multirow[b]{2}{*}{$\begin{array}{c}\text { Conservation } \\
\text { du lait }\end{array}$} & \multirow[b]{2}{*}{ Série } & \multicolumn{2}{|c|}{ Nombre de } & \multicolumn{5}{|c|}{ Pourcentage de } \\
\hline & & Echant. & $\begin{array}{l}\text { Colonies } \\
\text { prélevées }\end{array}$ & $\begin{array}{l}\text { Colonies } \\
\text { reprises }\end{array}$ & $\begin{array}{l}\text { Catalase* } \\
\text { négative }\end{array}$ & $\begin{array}{l}\text { S. lactis* } \\
\text { cremoris }\end{array}$ & $\begin{array}{l}\text { Strepto- } \\
\text { coques* } \\
\text { fécaux }\end{array}$ & $\begin{array}{l}\text { Leuco- } \\
\text { nostoc* }\end{array}$ \\
\hline Plus de $15^{\circ} \mathrm{C}$ & $\begin{array}{l}\text { I } \\
\text { II } \\
\text { III } \\
\text { IV }\end{array}$ & $\begin{array}{l}15 \\
23 \\
30 \\
22\end{array}$ & $\begin{array}{l}120 \\
184 \\
240 \\
176\end{array}$ & $\begin{array}{l}99 \\
92 \\
88 \\
95\end{array}$ & $\begin{array}{l}86 \\
97 \\
85 \\
86\end{array}$ & $\begin{array}{l}58 \\
66 \\
60 \\
47\end{array}$ & $\begin{array}{l}\text { ND } \\
1 \\
5,4 \\
4,0\end{array}$ & $\begin{array}{l}\text { ND } \\
5 \\
0 \\
17,6\end{array}$ \\
\hline Entre 8 et $10^{\circ} \mathrm{C}$ & $\begin{array}{l}\text { I } \\
\text { II } \\
\text { III } \\
\text { IV }\end{array}$ & $\begin{array}{r}19 \\
11 \\
11 \\
6\end{array}$ & $\begin{array}{r}152 \\
88 \\
88 \\
48\end{array}$ & $\begin{array}{r}100 \\
98 \\
90 \\
96\end{array}$ & $\begin{array}{l}89 \\
95 \\
81 \\
83\end{array}$ & $\begin{array}{l}50 \\
78 \\
67 \\
58\end{array}$ & $\begin{array}{l}\text { ND } \\
0 \\
0 \\
0\end{array}$ & $\begin{array}{c}\text { ND } \\
1 \\
0 \\
12,5\end{array}$ \\
\hline Moins de $5^{\circ} \mathrm{C}$ & $\begin{array}{l}\text { I } \\
\text { II } \\
\text { III } \\
\text { IV }\end{array}$ & $\begin{array}{r}13 \\
16 \\
5 \\
7\end{array}$ & $\begin{array}{r}104 \\
128 \\
40 \\
56\end{array}$ & $\begin{array}{r}100 \\
94 \\
88 \\
80\end{array}$ & $\begin{array}{l}90 \\
88 \\
78 \\
66\end{array}$ & $\begin{array}{l}61 \\
78 \\
65 \\
41\end{array}$ & $\begin{array}{l}\text { ND } \\
5 \\
0 \\
3,6\end{array}$ & $\begin{array}{l}\text { ND } \\
9 \\
0 \\
3,6\end{array}$ \\
\hline Total & & 178 & 1424 & 94 & 85 & 61 & - & - \\
\hline
\end{tabular}

*\% des colonies reprises ; ND : non déterminé. 

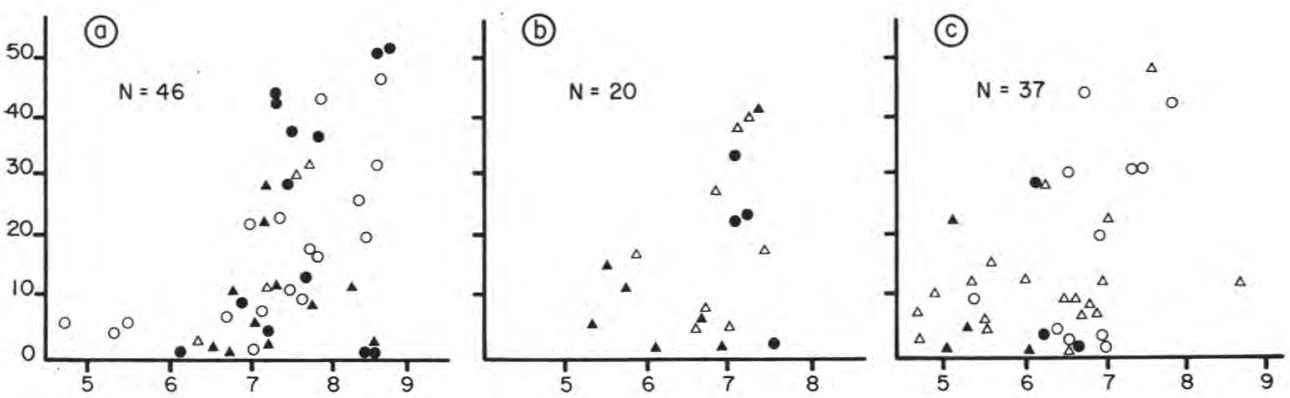

Fig. 5

Pourcentage de streptocoques lactiques par rapport à la flore totale (en ordonnée), en fonction du logarithme du nombre de germes totaux (en abscisse). Légende : voir figure 4.

Percentage of lactic streptococci (vertical axis) as a function of the total counts in milk (lg $10, a b s c i s s a)$. For further explanation see legend to Figure 4.

présent à un niveau appréciable seulement au cours de la $4^{\text {e }}$ série (à l'automne, après la rentrée des vaches en étable) et en proportions apparemment d'autant plus grandes que la température de conservation du lait à la ferme était plus élevée.

\section{Résultats des dénombrements}

a) Relation entre les nombres de micro-organismes résistant à la pénicilline et la flore totale

La figure 4 montre, quelle que soit la série ou l'origine des échantillons, que le nombre de micro-organismes résistant à la pénicilline dans le lait refroidi à moins de $12^{\circ} \mathrm{C}$ augmente lorsque la flore totale s'accroît. Par contre, pour le lait non refroidi cette relation est pratiquement inexistante.

On remarque également, pour une même période de l'année, que la flore totale du lait non refroidi est en général à un niveau plus élevé que dans les autres échantillons.

b) Proportion de bactéries lactiques dans la flore totale

Sur la figure 5 sont présentées les proportions de streptocoques lactiques dans la flore totale pour une période (série II) jugée comme étant plus favorable au développement de la flore lactique que les trois autres périodes. Cette proportion a été obtenue en multipliant les résultats de dénombrement des petites colonies par la fréquence moyenne des streptocoques lactiques dans ces dernières (tab. 3).

On constate que cette proportion n'est pas très importante, quelle que soit l'origine du lait ou son mode de conservation. En 
Fig. 6

Relation entre les logarithmes des nombres de streptocoques lactiques (en ordonnée) et de Pseudomonas (en abscisse).

Légende : voir figure 4.

Relationship between the num bers of lactic streptococci ( $\lg _{10}$, vertical axis) and the numbers of Pseudomonas in milk ( $\lg _{10}$, abscissa).

For further explanation see legend to Figure 4.
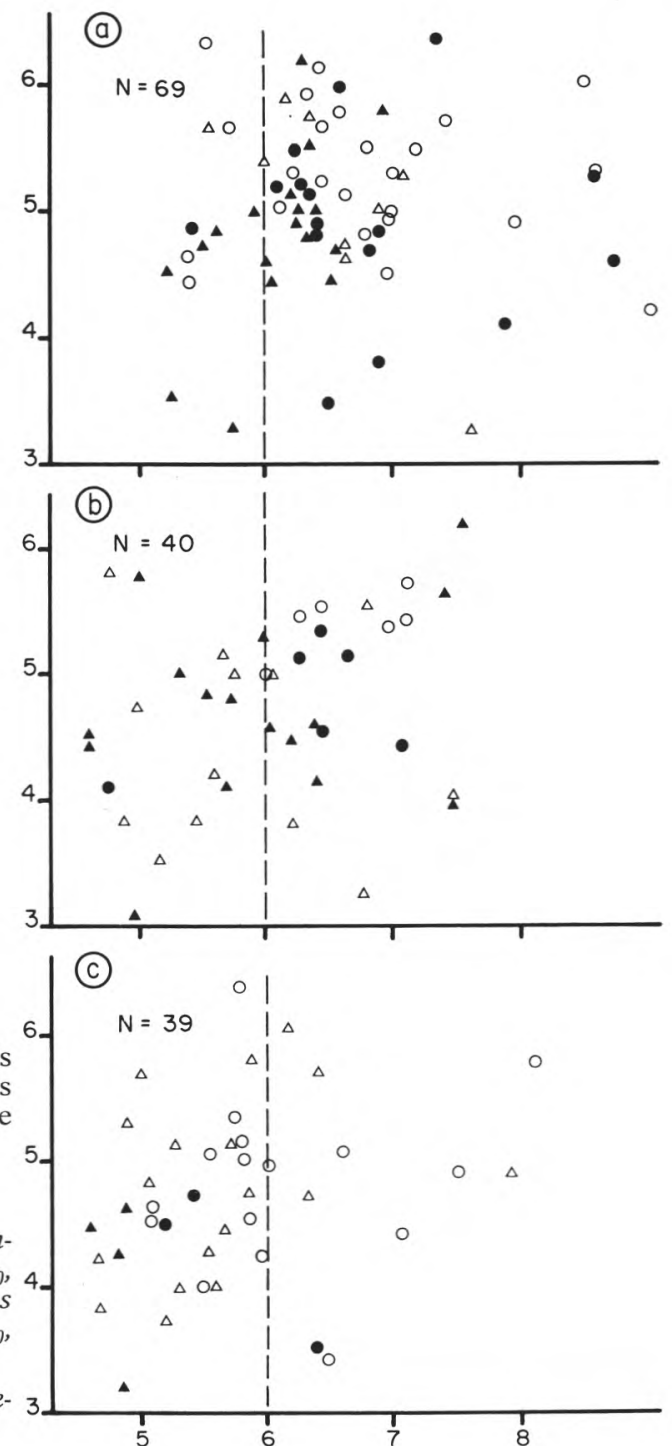

effet, dans la plupart des échantillons de lait refroidi la flore lactique ne représente pas plus de $30 \%$ de la flore totale. On remarquera cependant la présence de streptocoques lactiques à un niveau non négligeable (plus de $20 \%$ dans un certain nombre d'échantillons de lait refroidi. Dans les échantillons de lait non refroidi, ce pourcentage est plus important mais il ne dépasse néanmoins pas 50 . 
c) Relation entre la flore lactique et la flore pénicillino-résistante oxydase-positive (Pseudomonas)

Sur l'ensemble des résultats de l'étude (toutes séries confondues) on a étudié la relation entre le nombre de streptocoques lactiques (estimé à partir du nombre de petites colonies) et le nombre de Pseudomonas (estimé à partir du nombre de micro-organismes résistants à la pénicilline oxydase-positifs). La figure 6 illustre cette relation. On peut constater que le nombre de Pseudomonas du lait est indépendant du nombre de streptocoques lactiques, quelle que soit l'origine du lait ou son mode de conservation. Toutefois, les streptocoques lactiques sont généralement en nombres inférieurs à $1 \mathrm{million} / \mathrm{ml}$.

\section{DISCUSSION}

La flore résistante à la pénicilline était composée dans sa totalité de bactéries à Gram négatif. Cela ne veut pas dire que nous ayons énuméré de cette façon toutes les bactéries à Gram négatif du lait. Nous avons en effet trouvé, dans la flore sensible à la pénicilline, quelques bactéries coliformes et des bactéries pigmentées jaunes pouvant appartenir au groupe Flavobacter-Cytophaga. On sait, par ailleurs, que les Moraxella sont, par définition, sensibles à la pénicilline (Lautrop, 1974). Cependant, Vanderzant et Patel (1967), Thomas et Thomas (1973), Muir et al. (1979) et Richard (1981) ont montré, pour la flore psychrotrophe tout au moins, que les bactéries à Gram négatif du lait comprenaient une très faible proportion de représentants de ce groupe ou de ce genre. On peut donc dire que la numération de la flore résistante à la pénicilline correspond à peu près, pour le lait réfrigéré, à celle de la flore à gram négatif ; elle peut entraîner une sous-estimation de cette dernière lorsqu'on examine du lait non réfrigéré.

Moyennant cette réserve, les résultats que nous avons trouvés confirment, sur une base statistique beaucoup plus large, ceux de Thomas et al. (1962) et de Twoney et Crawley (1968) ; ils précisent également la nature ou donnent au moins des caractères technologiques de la flore dominante du lait cru très chargé en bactéries. En effet, on a constaté, comme ces auteurs, que dans le lait contenant plus de 1 million de micro-organismes par $\mathrm{ml}$, les streptocoques et les bactéries à Gram négatif constituaient l'essentiel de la flore. Cela s'explique par le fait que ces micro-organismes sont mieux adaptés à la culture dans le lait cru que les microcoques ou les bactéries corynéformes qui eux sont les constituants essentiels de la flore du lait à un niveau moindre de pollution. Ce sont également ces streptocoques et ces bactéries à Gram négatif qui dominent habituellement dans la vaisselle laitière mal lavée (Thomas et Thomas, 1977). C'est pourquoi sans doute, nous avons trouvé une 
assez forte proportion de streptocoques lactiques dans la flore totale du lait refroidi à moins de $5^{\circ} \mathrm{C}$, alors qu'ils ne se développent normalement pas à cette température.

Comme Muir et al. (1979) nous avons constaté, en été, une prédominance de bactéries coliformes sur les Pseudomonas, mais seulement dans des laits non refroidis, alors que cet auteur avait fait cette remarque pour le lait réfrigéré. Cette prédominance peut être expliquée par une croissance de bactéries coliformes plus rapides que celles des Pseudomonas lorsque la température du matériel de traite ou du lait remonte.

Par ailleurs, si la flore lactique avait joué un rôle antagoniste vis-à-vis des autres bactéries du lait, en particulier à l'égard des Pseudomonas, on aurait dû, comme conséquence, observer des nombres de ces derniers d'autant plus faibles que le niveau de la flore lactique était plus élevé. Ce n'est évidemment pas ce que nous avons constaté : la flore lactique et la flore nuisible (Pseudomonas et/ou bactéries coliformes) paraissent coexister jusqu'à un niveau élevé de population (respectivement jusqu'à $10^{6}$ et $10^{8}$ germes $/ \mathrm{ml}$ ).

Cela provient peut-être du fait que la flore lactique n'atteint pas un niveau suffisamment élevé pour entraîner une modification des propriétés du lait rendant défavorable la croissance des autres bactéries (acidification ou production de traces d'eau oxygénée (Juff et Babel, 1975).

On a tenté de réduire artificellement la multiplication de la flore nuisible du lait en éliminant de ce dernier, l'oxygène dissous indispensable à la croissance de bactéries aérobies strictes (Pseudomonas entre autres) ou plus naturellement, en l'inoculant avec des streptocoques lactiques (Juff et Babel, 1975). La première méthode ne semble pas avoir tenu ses promesses et la seconde, en plus de sa lourdeur, ne paraît pas être efficace quand le niveau de contamination du lait en bactéries à Gram négatif est élevé.

Il en résulte que si l'on veut maintenir la flore nuisible à un niveau où elle n'ait pas de conséquences sur la qualité des fromages, une amélioration de l'hygiène à la ferme (nettoyage correct de la vaisselle, refroidissement adéquat du lait) est nécessaire. C'est l'objectif du paiement du lait suivant sa qualité bactériologique.

\section{Bibliographie}

AbD-el-MaLeK (Y.) and Gibson (T.) (1948), - Studies in the bacteriology of milk. I. The streptocci ; II. The staphylococci and micrococci. J. Dairy Res., 15, 233-260.

AbD-EL-Malek (Y.) and Gibson (T.) (1952). - Studies in the bacteriology of milk. III. The corynebacteria of milk. J. Dairy Res., 19, 153-159.

ABD-EL-MALEK (Y.) and GiBSon (T.) (1952). - Studies in the bacteriology of milk. IV. The Gram-negative rods of milk. J. Dairy Res., 19, 294-301.

Benzecri (J. P.) (1975), - L'analyse des données. La Taximonie. Dunod, Paris. 
Colwell (R. R.) and Wiebe (W. J.) (1970). - " Core " characteristics for use in classiffying aerobic, heterotophic bacteria by numerical taxonomy. Bull. Georgin Acad. Sci., 28, 165-185.

Corlett Jr. (D. A.), Lee (J.S.) and Sinnhuber (R. O) (1965). - Application of replica plating and computer analysis for rapid identification of bacteria in some foods. Appl. Microbiol., 13, 808-817.

CowAN (S. T.) (1974), - Gram-negative facultatively anaerobic rods. In «Bergey's Manual of Determinative Bacteriology» edited by Buchanan R. E. and Gibbons, N.E., The Williams and Wilkins Company, Baltimore (U.S.A.).

GABY (W. L.) and HadLeY (C.) (1957). - Practical laboratory test for the identification of Pseudomonas aeruginosa., J. Bact., 74, 356-358.

Gilchrist (J.E.), Campbell (J. E.), Donnelly (C. B.), Peeler (J. T.) and Delaney (J. M.) (1973). - Spiral plate method for bacterial determination. Appl. Microbiol., 25, 244-252.

GyllenBeRg (H.) (1963). - A general method for deriving determination schemes for random collections of microbial isolates. Ann. Acad. Scient. Fen. Ser. A, IV Biologica, 69.

Harrigan (W. F.) and McCance (M. E) (1976), - Laboratory methods in food and dairy microbiology. Academic Press, London.

JufFs (H. S.) and BABEL (F. J.) (1975). - Inhibition of psychrotrophic bacteria by lactic cultures in milk stored at low temperature. J. Dairy Sci., 58, 1612-1619.

LAutrop (H.) (1974). - Gram-negative cocci and cocobacilli. Genus IV Acinetobacter in "Bergey's Manual of Determinative Bacteriology» edited by Buchanan (R.E.) and Gibbons (N.E.). The Williams and Wilkins Company, Baltimore (U.S.A.).

LowIS (M. J.) (1969). - A comparison of methods used to determine mobility of strains of Gram-negative bacteria isolated from aqueous pharmacentical preparations. Lab. Pract., 18, 929-932.

Mourgues (R.), Accolas (J. P.) et Auclair (J.) (1967). - L'utilisation du lait réfrigéré en tanks de ferme pour la fabrication du fromage. Fabrication du fromage de gruyère. Revue Laitière Française, 243, 289-298.

Muir (D. D.), Phillips (J. D.) and Dalgleish (D. G.) (1979). - The lipolytic and proteolytic activity of bacteria isolated from blended raw milk. J. Soc. Dairy Technol., 32, 19-23.

Patel (G. B.) and Blankenagel (G.) (1972). - Bacterial counts of raw milk and flavor of the milk after pasteurization and storage. J. Milk Food Technol., 35, 203-206.

PARK (R. W. A.) and Holding (A. J.) (1966). - Identification of some common Gram-negative bacteria. Lab. Pract., 15, 1124.

Picket (M. J.) and Pedersen (M. M.) (1968). - Screening procedure for partial identification of non-fermentative bacilli associated with man. Appl. Microbiol., 16, 1631-1632.

Punch (J. D.), Olson jr. (J. C.) and Thomas (E. L.) (1965). - Psychrophilic bacteria. III. Population levels associated with flavor or physical change in milk. J. Dairy Sci., 48, 1179-1183.

RicharD (J.) (1980). - Influence de l'agitation du lait cru sur les résultats de dénombrement de sa flore totale à l'aide d'une anse calibrée. Le Lait, 60, 211-225.

RICHARD (J.) (1981a). - Influence de diverses méthodes de nettoyage des machines à traire sur la "qualité de conservation " du lait cru à basse température. Le Lait, 61, 354-369.

RICHARD (J.) (1981b). - Classification adansonienne et identification de Pseudomonas psychrotrophes isolés du lait cru conservé à basse température. Ann. Microbiol.'(Inst. Pasteur), 132 A, 171-182. 
RICHARD (J.) (1981c). - Microbiological aspects of cold cleaning with an iodophor of milk pipeline installation. J. Appl. Bacteriol., 50, 229-238.

RICHARD (J.) (1982). - Dénombrement sélectif des bactéries du lait en fonction de la taille des colonies à l'aide d'un compteur électronique à laser. Sciences des Aliments, 2, 31-40.

Sandine (W. E.), Radich (P. C.) and EllikeR (P. R.) (1972). - Ecology of the lactic Streptococci. A review. J. Milk Food Technol., 35, 176-185.

SoKal (R. R.) and Sneath (P. H. A.) (1963). - Principles of numerical taxonomy, W. H. Freeman and Co., London.

Stanier (R. Y.), Palleroni (N. J.) and Doudoroff (M.) (1966). - The aerobic Pseudomonas: a taxonomic study. J. Gen. Microbiol., 43, 159-271.

Thomas (S. B.), Hobson (P. M.), Bird (E. R.), King (K. P.), Druce (R. G.) and Cox (D. R.) (1962). - The microflora of raw milk as determined by plating on yeastrel-milk agar incubated at $30^{\circ}$. J. Appl. Bacteriol., 25, 107-115.

Thomas (S. B.) and Thомаs (B. F.) (1973). - Psychrotrophic bacteria in refrigerated bulk-collected raw milk. Part 1 and 2. Dairy Ind., 38, 11-15, 61-70.

Thomas (S. B.) and Thomas (B. F.) (1977). - The bacterial content of milking machines and pipeline milking plants. Dairy Ind. Intern., 42, 19-25.

ThorNley (M. J.) (1963). - The differentiation of Pseudomonas from the other gram-negative bacteria on the basis of arginine metabolism. J. Appli. Bacteriol., 23, 37-52.

Twomey (A.) and Crawley (W. E.) (1968). - The microflora of milk, N.Z.J. Dairy Technol., 3, 44-48.

Vanderzant (C.) and Patel (G. D.) (1967). - Quantitative microbiol examination of some milks and meat products during storage by a replica plating technique. J. Milk Food Technol., 30, 171-178.

VASSAL (L.) et Auclair (J.) (1966). - L'utilisation du lait réfrigéré en tanks de ferme pour la fabrication du fromage. 1. Fabrication de fromages à pâte molle. Rev. Lait. Fr., 237, 666-673.

WitTer (L. D.) (1961). - Psychrotrophic bacteria. A Review. J. Dairy Sci., 44, 983-1015. 